\title{
Peroxidative metabolism of arachidonic acid in the course of Lyme arthritis
}

\author{
Wojciech Łuczaj', Anna Moniuszko², Magdalena Rusak', Joanna Zajkowska², \\ Sławomir Pancewicz' ${ }^{2}$, Elżbieta Skrzydlewska' \\ ${ }^{1}$ Department of Analytical Chemistry, Medical University of Bialystok, Poland \\ ${ }^{2}$ Department of Infectious Diseases and Neuroinfection, Medical University of Bialystok, Poland
}

Łuczaj W, Moniuszko A, Rusak M, Zajkowska J, Pancewicz S, Skrzydlewska E. Peroxidative metabolism of arachidonic acid in the course of Lyme arthritis. Ann Agric Environ Med. 2015; 22(3): 433-437. doi: 10.5604/12321966.1167708

\begin{abstract}
Objective. The objective of the study was measurement of serum arachidonic acid level as well as the product of its peroxidation - 8-isoPGF ${ }_{2 \alpha^{\prime}}$ and the activity of phospholipase $A_{2}$ and PAF-acetylhydrolase that participate in releasing 8 -isoPGF ${ }_{2 \alpha}$ from glycerol skeleton, and the potential designation of their role in the pathomechanism of Lyme disease (LD).

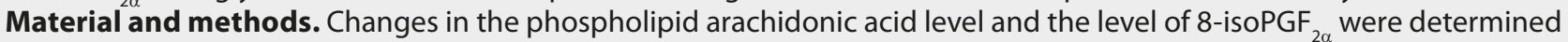
in the plasma and urine of patients with LA $(n=57)$ and of healthy controls $(n=41)$. The activity of phospholipase $A_{2}$ and PAF acetylhydrolase were assayed. All examined parameters were also measured in the plasma of some LA patients $(n=13)$ after antibiotics treatment.

Results. An almost 3-fold higher level of the total plasma 8-isoPGF $2 \alpha$ was observed in LA patients compared to the controls,

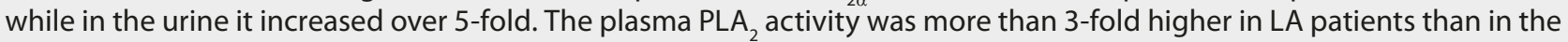
healthy subjects, while PAF acetylhydrolase activity was observed to be modestly, but not significantly lower. The total 8 -isoPGF ${ }_{2 \alpha}$ level in the plasma and urine of LA patients was significantly lower after antibiotics treatment. The plasma activity of PAF-AH in the LA patients was increased, while the CPLA ${ }_{2}$ activity decreased after antibiotics treatment.

Conclusions. It may be suggested that in the course of LA, the level of binding 8 -isoPGF ${ }_{2 \alpha}$ is significantly enhanced, and it may also be suggested that uncontrolled changes in the lipid status of some patients may make their Lyme arthritis unresponsive to antibiotics.
\end{abstract}

\section{Key words}

Lyme arthritis, isoprostanes, lipid peroxidation

\section{INTRODUCTION}

Lyme disease (LD) is a consequence of infection with the spirochete Borrelia burgdorferi, which is transmitted to humans by the feeding of infected Ixodes ticks [1]. The spirochete initially establishes infection in the skin and next disseminates to other organs, such as the heart, joints and nervous system [1]. B. burgdorferi contributes to the local tissue inflammation, which can manifest as arthritis. Arthritis develops over months after infection, affecting $60 \%-80 \%$ of untreated patients [2]. Lyme arthritis (LA) is characterized by tendonitis and synovitis. B. burgdorferi induces expression of $\beta 2 / C D 18$ integrins, which mediate the migration and adhesion of neutrophils and other leukocytes to the endothelium necessary for extravasation during inflammation, and are crucial for controlling the spirochete infection [3]. Moreover, $\beta 2$ integrin receptors are critically involved in neutrophil activation at the site of inflammation and their activation contributes to oxidative burst. Both of these pathways lead to over-generation of reactive oxygen species (ROS) and reactive nitrogen species (RNS), especially superoxide anion and nitrogen oxide [4]. The oxidative burst is considered to be one of the main antimicrobial neutrophil functions; however, the oxidative burst participates not only in the local killing of spirochetes. Enhanced generation of

Address for correspondence: Anna Moniuszko, Department of Analytical Chemistry, Medical University of Bialystok, Mickiewicza 2d, 15-222 Bialystok, Poland E-mail: annamoniuszko@op.pl

Received: 18 May 2013; accepted: 31 July 2014
ROS and changes in antioxidant abilities of patients observed in B. burgdorferi infection lead to oxidative stress formation [5]. In consequence, cellular components such as lipids, DNA and proteins are oxidatively modified, which leads to changes in their functions and contributes significantly to the pathology.

Lipids are particularly susceptible to attack of ROS, which results in the formation of a number of peroxidation products. The main components of lipids particularly susceptible to the action of ROS are polyunsaturated fatty acids, with commonly occurring arachidonic acid in particular. One group of oxidation products that may exert toxic effects on the cells are very reactive aldehydes, such as malondialdehyde and 4-hydroxynonenal, the level of which is also enhanced during LA [6]. Their generation and reactions with proteins disturb the structure and functions of biomembrane components, as well as cytosolic protein participating in the cellular signaling. Another class of lipid peroxidation products is the prostaglandin F2-like compounds group isoprostanes, mainly 8 -isoPGF ${ }_{2 \alpha} .8$-isoPGF $2 \alpha$ are produced in vivo independently of cyclooxygenases enzymes, primarily by free radical induced peroxidation of arachidonic acid [7]. It has been shown that the levels of 8 -isoPGF ${ }_{2 \alpha}$ are increased in a number of human diseases, including LA [8]. Measurement of 8 -isoPGF ${ }_{2 \alpha}$ is currently recognised as the most accurate to assess the oxidative injury in vivo; moreover, they are more stable than aldehydes generated during lipid peroxidation. In addition, products of the 8 -isoPGF ${ }_{2 \alpha}$ pathway have been found to exert potent biological actions and therefore may be pathophysiologic mediators of diseases [9]. 


\section{OJECTIVE}

The objective of this study was measurement of the serum arachidonic acid level as well as the product of its peroxidation - 8-isoPGF ${ }_{22}$, and the activity of phospholipase $\mathrm{A}_{2}$ and PAF-acetylhydrolase that participate in releasing of 8-isoPGF from glycerol skeleton and the potential designation of their role in the pathomechanism of LA.

\section{MATERIALS AND METHOD}

The main examined group (group I) consisted of 57 patients with LA (18 females, 39 males) before antibiotic treatment, aged 23-72 years (mean: - 49 years). Group II consisted of 13 patients with LA ( 2 females, 11 males) aged 23 to 76 years (mean: 51 years), treated with doxycyclinum or cefrixone for one week. The control group consisted of 41 healthy individuals (13 females, 28 males) aged 26-67 years (mean: 46 yearsd). The healthy controls were specifically screened for any sort of inflammatory process and for use of non-steroidal anti-inflammatory drugs.

Diagnosis of LA was confirmed by epidemiological anamnesis, clinical picture - arthritis caused by B. burgdorferi and serological examinations results - detection of anti/ B. burgdorferi IgM and IgG antibodies in ELISA method (Borrelia recombinant IgG and IgM, Biomedica, Austria) and at least a 4-times increase of their concentration in IgG class. In all cases, results of ELISA test were confirmed by Western blot testing (DRG Diagnostics).

In epidemiological anamnesis,s $90 \%$ of patients reported previous tick bites. In all cases, pain limiting movement of big joints (especially knee, elbow, shoulder) and muscles, especially of thighs and shoulders, were observed.

In all cases, other concomitant inflammatory processes were excluded. In treatment, all the patients concomitantly were taking non-steroidal anti-inflammatory drugs.

Blood was collected into EDTA tubes from $57 \mathrm{LA}$ patients before antibiotic treatment for the arthritis, as well as from healthy controls. Additionally, blood was collected from 13 LA patients before antibiotic treatment as well as after one week treatment with doxycyclinum or ceftriaxone. The blood was centrifuged at $3,000 \times \mathrm{g}\left(4^{\circ} \mathrm{C}\right)$ to obtain plasma. Urine was also collected from the same patients.

The study was undertaken with the permission of the Bioethical Committee of the Medical University of Bialystok, Poland, after obtaining the written informed consent of the patients who participated in the study.

Determination of 8-isoPGF ${ }_{2 \alpha}$ level. The 8 -isoPGF ${ }_{2 \alpha}$ level was determined in the plasma as well as in the urine using the LC-MS $\backslash$ MS method $[10,11]$. The plasma 8 -isoPGF ${ }_{2 \alpha}$ were determined as free 8-isoPGF ${ }_{2 \alpha}$ - existing in the organism as not-esterified in phospholipids form (hydrolyzed in organism), and as total 8-isoPGF ${ }_{2 \alpha}$, containing free - not esterified and esterified in phospholipids 8 -isoPGF ${ }_{2 \alpha}$. To determine total 8 -isoPGF ${ }_{2 \alpha} 1.0 \mathrm{ml}$ of plasma was thawed and homogenized with $30 \mu$ linternal standard (300 pg 8-isoPGF $\left.-\mathrm{d}_{4}\right), 80 \mu \mathrm{l}$ of a solution containing BHT $(10 \mathrm{mM})$ and triphenylphosphine $(1 \mathrm{mM})$ in ethanol, and the mixture was thoroughly vortexed. Next, $400 \mu \mathrm{l}$ of a solution containing $25 \%(\mathrm{w} / \mathrm{v}) \mathrm{KOH}$ was added and the mixture heated in a water bath for 45 minutes at $40^{\circ} \mathrm{C}$ in order to hydrolyse the esterified isoprostanes. After hydrolysis, the sample was diluted with $3 \mathrm{ml}$ of water, in order to prevent protein precipitation, followed by vortexing. Acetic acid was added to $\mathrm{pH} 3$ to prevent dissociation of the isoprostanes. A solid phase column (tC18 Sep-pak Vac, $3 \mathrm{ml}, 200 \mathrm{mg}$; Waters Chromatography) was conditioned with $3 \mathrm{ml}$ of methanol, followed by $3 \mathrm{ml}$ of water that was adjusted to $\mathrm{pH} 3$ with glacial acetic acid. The sample was vortexed again and loaded onto the SPE column. The testtube was washed with $3 \mathrm{ml}$ of water ( $\mathrm{pH} 3$, corrected with glacial acetic acid) and the washing solution also loaded into the column and the cartridge dried. The cartridge was then washed with $3 \mathrm{ml}$ of heptane to remove lipids. The cartridges were dried again under vacuum, and the sample was again eluted with $7 \mathrm{ml}$ of eluent consisting of ethyl acetate/heptane/ methanol (50/40/10\%). The resulting solution was evaporated to dryness under a gentle stream of nitrogen. The residue was dissolved in $60 \mu \mathrm{l}$ acetonitrile/water (60/40\% with $0.05 \%$ (v/v) acetic acid). For quantification of free 8-isoPGF $2 a$ in plasma, the same protocol was applied but an alkaline hydrolysis step was omitted.

To urine, 8-isoPGF ${ }_{2 \alpha}$ determination urine samples $(1 \mathrm{ml})$, were added to $0.5 \mathrm{ml}$ of $15 \% \mathrm{KOH}$ and mixed and allowed to stand for $30 \mathrm{~min}$. The $\mathrm{pH}$ was next adjusted with $6 \mathrm{M} \mathrm{HCl}$ to 2.5. The sample was then subjected to solid phase extraction (SPE). BondElut LMS polymer $100 \mathrm{mg}$ SPE cartridges were used (purchased from Varian). The SPE cartridge was conditioned with $1 \mathrm{ml}$ of ethanol and $1 \mathrm{ml}$ of Sorensen buffer. The sample was applied to the cartridge, which was then sequentially washed with $1 \mathrm{ml}$ each of Sorensen buffer and $5 \%$ ethanol in water. The analyte and internal standards were eluted from the cartridge by using $1 \mathrm{ml}$ of ethanol. The eluate was collected and dried under a gentle stream of nitrogen. The resulting residue was then reconstituted with $100 \mu \mathrm{l}$ of $20 \%$ acetonitrile. A Phenomenex RP C18 column $(150 \times 2 \mathrm{~mm}, 3 \mu \mathrm{m})$ was used for chromatographic analysis. A gradient separation was performed, starting with a solvent that consisted of $60 \%$ water ( $\mathrm{pH} 3.85$ ) and $40 \%$ acetonitrile running for 4 minutes. From 4-5 minutes, a gradient was run to $100 \%$ acetonitrile, which ran for 8 minutes. From $8-9$ minutes, a gradient was used to return to the original eluent composition and the system was equilibrated for 15 minutes. The flow rate was $200 \mu \mathrm{l} / \mathrm{min}$, the temperature column was set at $30^{\circ} \mathrm{C}$; injection volume $10 \mu \mathrm{l}$. The following parameters were employed throughout all MS experiments for ESI with negative ion polarity. The target ions m/z 353, EIC: m/z 193 and 255 , the fragments detected over a scan range of $\mathrm{m} / \mathrm{z}$ $150-400$.

Determination of phospholipid fatty acid level. The plasma level of phospholipid fatty acids was analyzed by GC with a FID detector [12]. First, the lipids were isolated by Folch extraction using chloroform/methanol mixture $(2: 1, \mathrm{v} / \mathrm{v})$ in the presence of $0.01 \%$ butylated hydroxytoluene (BHT). TLC separated phospholipids fatty acids with the mobile chase heptane - diisoprophyl ether - acetic acid (60:40:3, $\mathrm{v} / \mathrm{v} / \mathrm{v}$ ) and transmetylated to fatty acid methyl ester (FAME) with boron trifluoride in methanol reagent under nitrogen atmosphere at $100^{\circ} \mathrm{C}$ for $30 \mathrm{~min}$ for phospholipids. The FAMES were analyzed by gas chromatography with a FID (flame ionization detector) using a capillary column coated with CP-Sil 88 stationary phase. The injector and FID detector temperatures were kept at $260^{\circ} \mathrm{C}$. The column temperature was programmed from $150^{\circ} \mathrm{C}(2 \mathrm{~min})-230^{\circ} \mathrm{C}(10 \mathrm{~min})$ at 
$4^{\circ} \mathrm{C} / \mathrm{min}$. Identification of FAMEs was made by comparison with their retention time with standards.

Changes in cPLA 2 and PAF-AH activities. Commercial assay kits were used to determine cPLA, and PAF-AH activities (Cayman, Ann Arbor, MI, USA).

Statistical analysis. Data obtained in the study are expressed as mean \pm SD. These data were analyzed by standard statistical analyses, one-way analysis of variance (ANOVA) with Tukey's test, to determine significant differences between groups. A $p$ value of $<0.05$ was considered significant.

\section{RESULTS}

Oxidative damage was implicated in the pathogenesis of LD. The level of free AA in the plasma was observed to be significantly higher (by about 22\%) in patients with LA, compared to controls, while the esterified AA level was decreased (by about 35\%) (Tab. 1).

Table 1. Level of arachidonic acid [AA], isoprostanes [8-isoPGF ], activity of phospholipase $\mathrm{A}_{2}\left[\mathrm{CPLA}_{2}\right]$ and acetylhydrolase PAF [PAF-AH] in Lyme arthritis patients and the control group

\begin{tabular}{|c|c|c|}
\hline Parameters & $\begin{array}{l}\text { Control } \\
{[n=41]}\end{array}$ & $\begin{array}{l}\text { Lyme arthritis } \\
\qquad[\mathrm{n}=57]\end{array}$ \\
\hline $\begin{array}{l}\text { Plasma free AA level } \\
{[\mathrm{nmol} / \mathrm{ul}]}\end{array}$ & $0.166 \pm 0.026$ & $0.203 \pm 0.065^{\mathrm{a}}[22 \%]$ \\
\hline $\begin{array}{l}\text { Plasma phospholipid AA level } \\
{[\mathrm{nmol} / \mathrm{ul}]}\end{array}$ & $36.86 \pm 6.32$ & $24.11 \pm 6.36^{\mathrm{a}}\left[35 \%^{-}\right]$ \\
\hline $\begin{array}{l}\text { Plasma total 8-isoPGF }{ }_{2 \alpha} \text { level } \\
{[\mathrm{pg} / \mathrm{ml}]}\end{array}$ & $18.99 \pm 5.09$ & $52.15 \pm 25.17^{\mathrm{a}}[275 \%]$ \\
\hline $\begin{array}{l}\text { Plasma free 8-isoPGF }{ }_{2 \alpha} \text { level } \\
{[\mathrm{pg} / \mathrm{ml}]}\end{array}$ & $5.16 \pm 1.64$ & $3.48 \pm 1.66^{\mathrm{a}}\left[33 \%^{-}\right]$ \\
\hline$\%$ of free 8-isoPGF ${ }_{2 \alpha}$ level & $27 \%$ & $7 \%$ \\
\hline $\begin{array}{l}\text { Urine 8-isoPGF }{ }_{2 \alpha} \text { level } \\
\text { [pg/mg creatinine] }\end{array}$ & $37.04 \pm 11.01$ & $\begin{array}{c}206.59 \pm 137.55^{\mathrm{a}} \\
\quad[558 \%]\end{array}$ \\
\hline $\begin{array}{l}\text { Plasma PLA }{ }_{2} \text { activity } \\
{[\mathrm{nmol} / \mathrm{min} / \mathrm{ml}]}\end{array}$ & $9.00 \pm 1.44$ & $28.68 \pm 11.2^{\mathrm{a}}[319 \%]$ \\
\hline $\begin{array}{l}\text { Plasma PAF-AH activity } \\
{[\mathrm{nmol} / \mathrm{min} / \mathrm{ml}]}\end{array}$ & $25.65 \pm 3.96$ & $24.17 \pm 8.87\left[6 \%{ }^{-}\right]$ \\
\hline
\end{tabular}

${ }^{a}$ statistically significant differences for $\mathrm{p}<0.05$

Although the level of total 8-isoPGF ${ }_{2 \alpha}$ was significantly higher in LA patients compared to the controls, the increase in this isoprostane in the plasma was smaller than in the urine. An approximately 3 -fold higher level of the total plasma 8-isoPGF ${ }_{2 \alpha}$ was observed in LA patients compared to the controls, while in the urine it increased over 5-fold. However, the level of free 8-isoPGF ${ }_{2 \alpha}$ in the control plasma was $27 \%$ of the total level, while the level of free 8 -isoPGF in the plasma of LA patients was 7\% of the total level. Platelet activating factor-acetylhydrolase activity in the plasma was observed to be slightly, but not significantly lower (by about $6 \%$ in LA patients compared to the controls. By contrast, the plasma cPLA activity was over 3 -fold higher in the LA patients than in the healthy subjects.

All examined parameters were also measured in the plasma of the LA patients after antibiotics treatment (Tab. 2).

No significant differences were observed in the levels of free and esterified AA before and after treatment. However,
Table 2. Level of arachidonic acid [AA], isoprostanes [8-isoPGF ${ }_{22}$, activity of phospholipase $\mathrm{A}_{2}\left[\mathrm{CPLA}_{2}\right]$ and acetylhydrolase PAF [PAF-AH] in Lyme arthritis patients plasma and urine before and after antibiotics treatment

\begin{tabular}{|c|c|c|}
\hline \multirow[b]{2}{*}{ Parameters } & \multicolumn{2}{|c|}{ Lyme arthritis [n=13] } \\
\hline & $\begin{array}{c}\text { Before antibiotic } \\
\text { treatment }\end{array}$ & $\begin{array}{l}\text { After antibiotic } \\
\text { treatment }\end{array}$ \\
\hline $\begin{array}{l}\text { Plasma free AA level } \\
{[\mathrm{nmol} / \mathrm{ul}]}\end{array}$ & $0.190 \pm 0.048$ & $0.180 \pm 0.036\left[5 \%{ }^{-}\right]$ \\
\hline $\begin{array}{l}\text { Plasma phospholipid AA level } \\
\text { [nmol/ul] }\end{array}$ & $20.06 \pm 3.56$ & $21.18 \pm 3.45[6 \%]$ \\
\hline $\begin{array}{l}\text { Plasma total 8-isoPGF }{ }_{2 \alpha} \text { level } \\
{[\mathrm{pg} / \mathrm{ml}]}\end{array}$ & $75.43 \pm 39.87$ & $48.56 \pm 21.51^{\mathrm{a}}\left[27 \%^{-}\right]$ \\
\hline $\begin{array}{l}\text { Plasma free 8-isoPGF }{ }_{2 \alpha} \text { level } \\
{[\mathrm{pg} / \mathrm{ml}]}\end{array}$ & $3.01 \pm 1.23$ & $2.83 \pm 1.02\left[6 \%{ }^{-}\right]$ \\
\hline$\%$ of free 8 -isoPGF ${ }_{2 \alpha}$ level & $4 \%$ & $6 \%$ \\
\hline $\begin{array}{l}\text { Urine 8-isoPGF }{ }_{2 \alpha} \text { level } \\
{[\mathrm{pg} / \mathrm{mg} \text { creatinine] }}\end{array}$ & $237.42 \pm 132.14$ & $144.24 \pm 83.21^{\mathrm{a}}\left[39 \%{ }^{-}\right]$ \\
\hline $\begin{array}{l}\text { Plasma PLA activity } \\
{[\mathrm{nmol} / \mathrm{min} / \mathrm{ml}]}\end{array}$ & $31.92 \pm 7.62$ & $23.51 \pm 3.21^{\mathrm{a}}\left[26 \%{ }^{-}\right]$ \\
\hline $\begin{array}{l}\text { Plasma PAF-AH activity } \\
{[\mathrm{nmol} / \mathrm{min} / \mathrm{ml}]}\end{array}$ & $23.14 \pm 4.72$ & $29.85 \pm 6.58^{\mathrm{a}}[29 \%]$ \\
\hline
\end{tabular}

${ }^{a}$ statistically significant differences for $p<0.05$

the total 8 -isoPGF ${ }_{2 \alpha}$ level patients were significantly lower (by about $27 \%$ and $39 \%$, respectively) in the plasma and the urine of LA patients after antibiotics treatment. There were no significant differences in the plasma level of free 8 -isoPGF $2 \alpha$ before and after antibiotics treatment. The plasma concentration of free 8 -isoPGF - $_{2 \alpha}$ before and after the treatment was $7 \%$ and $6 \%$ of the total amount, respectively. Surprisingly, the plasma activity of PAF-AH in the LA patients increased (by about 29\%), while the cPLA 2 activity decreased (by about 26\%) after antibiotics treatment.

\section{DISCUSSION}

LD caused by infection with the spirochete $B$. burgdorferi is the most common vector-borne disease in Europe and North America. It has been proved that during B. burgdorferi infection, ROS generation is enhanced, while the antioxidant abilities, particularly the level of vitamin E, which acts as an efficient antioxidant in the lipid phase of the biomembranes, are decreased [13]. In such a situation, ROS may react with polyunsaturated fatty acids, among which, arachidonic acid is commonly found. When it is attacked by reactive species, peroxidation products are formed. As first, lipid peroxides and low molecular aldehydes are produced. An elevated level of low molecular phospholipid peroxidation products in the course of LA was revealed earlier [6]. The relatively stable group of lipid peroxidation products are prostaglandinlike compounds - isoprostanes that are also significantly enhanced during the course of LA, as has been previously shown and in this study.

Isoprostanes belong to the eicosanoids family that comprise a diverse class of over 100 bioactive lipid mediators derived from the metabolism of polyunsaturated fatty acids by enzymatic as well as non-enzymatic pathways. Analysis of joints from LA-resistant and susceptible mouse strains revealed not only COX catalyzing generation of $\mathrm{PGE}_{2}$ and $\mathrm{PGD}_{2}$, but also the production of eicosanoids, including products of CYP and 12-LOX - 11,12-EEET and $\mathrm{PD}_{1}$, 
respectively. However, the lipidomic analysis of joints from COX-2-deficient animals during infection with $B$. burgdorferi showed reduction in the products of COX and LOX action [14]. Moreover, it was shown that mice lacking COX-2 are characterized by sustained neutrophil influx into the joints and, in consequence, inflammatory response and prolonged arthritis pathology [15]. In such a situation, the non-enzymatic mechanisms of oxidation of arachidonic acid esterified at the $s n-2$ position of cellular and lipoprotein phospholipids may play an important role. Arachidonoyl phospholipid peroxides may rearrange to a series of regioand stereoisomers of eicosanoid mediators, including the $\mathrm{PGF}_{2}$-like compounds termed 8-isoPGF ${ }_{2 \alpha}[16]$. It was proved

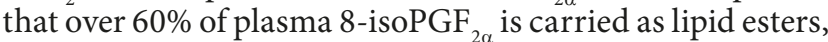
with the remainder circulating as free 8 -isoPGF ${ }_{2 \alpha}$ [10], which is confirmed in the presented study. In consequence, the level of arachidonic acid is decreased, as shown in the current study.

The elevated level of 8 -isoPGF has $_{2 \alpha}$ been observed in a number of human diseases that have been linked to oxidative stress, and this study has confirmed that LA is accompanied by a 3 -fold increase in the level of esterified 8 -isoPGF $\mathrm{in}_{2 \alpha}$ in the plasma, while the level of the free form of isoprostanes has a tendency to decrease. The results of the current study indicate that in the course of LD formation of 8-isoPGF ${ }_{2 \alpha}$ was enhanced faster than their hydrolyzing from phospholipids.

A unique aspect of the genesis of isoprostanes compared with prostaglandins formed as products of the cyclooxygenase, is that isoprostanes are initially generated in phospholipids form and are subsequently released as a free form. The mechanism responsible for releasing these mediators after generation in membrane phospholipids is largely unknown. 8-isoPGF ${ }_{2 \alpha}$ are realized by phospholipases, mainly by phospholipases $\mathrm{A}_{2}^{2 \alpha}$ - enzymes hydrolyzing a bond at the sn-2 position of the glycerol phospholipids. Released isoprostanes are subsequently excreted into the urine [17]. In the presented study, the enzymatic activity of phospholipases $\mathrm{A}_{2}$ was higher in the plasma of the LA patients compared to the controls. This may explain the increase in $\mathrm{F}_{2}$-IsoPs in the urine, which was also reported earlier [6], resulting in an accumulation of esterified $\mathrm{F}_{2}$-IsoPs in the lipids [18]. It was shown that $B$. burgdorferi induced cytosolic phospholipase $\mathrm{A}_{2}$ mRNA level in the joints of infected mice [15]. The expression of both phospholipases $\mathrm{A}_{2}$ and COX-2 was observed during $B$. burgdorferi infection, and their temporal expression altered by COX-2 enzyme inhibition suggested a potential feedback mechanism for the regulation of mRNA for these enzymes by the products of COX-2. Separation of arachidonic acids by phospholipase $A_{2}$ also indirectly contributes to increased ROS generation because the further arachidonic acid metabolism is partially connected with COX and LOX action in which ROS generation is observed [19]. Thus, phospholipase $\mathrm{A}_{2}$ may also enhance lipid peroxidation. Enhancement of the phospholipase $\mathrm{A}_{2}$ activity by the oxidative stress is accompanied by a decrease in the level of vitamin E and selenium. There is a cross-talk between two different fatty acids oxidation pathways. The COXs are implicated in 8-isoPGF ${ }_{2 \alpha}$ formation because COX action leads to the generation of ROS that can nonenzymatically oxidize arachidonic acids, whereas lipid hydroperoxides can activate the COXs. It must be emphasized that phospholipase $\mathrm{A}_{2}$ may also reveal its protective action by enhancing lipid hydroperoxide metabolism, as well as by repairing the biological membranes removing the damaged lipids [20].

It was also found that among phospholipases, intracellular and plasma PAF acetylhydrolases also have a high affinity for hydrolyzing oxidatively modified phospholipids and release isoprostanes localized at sn- 2 position of glycerol [21]. However, the rate of hydrolysis of isoprostanes is much lower compared with that of other substrates utilized by these enzymes. In the presented study, an increase in PAF$\mathrm{AH}$ activity in the plasma of the LA patients was observed, compared to the controls. Because this enzyme is susceptible to oxidative inactivation, it is possible that certain lipid oxidation products generated during LA may inhibit it. The diminished activity of plasma PAF acetylhydrolase and the high level of the esterified form of isoprostanes in LA confirm this suggestion. Moreover, the increase in the activity of PAF acethylhydrolase was observed after antibiotics treatment, when the level of isoprostanes was diminished. Independently of the above suggestion, the decrease in the plasma PAF acetylhydrolase activity during some other diseases, such as asthma, atopy, stroke or Parkinson disease, was also shown $[8,22]$.

These studies indicate two different roles of isoprostanes and phospholipases in the physiology and Lyme arthritis pathology. In physiological conditions, phospholipase $\mathrm{A}_{2}$ and PAF acetylhydrolase modulate cellular homeostasis considering the abilities to initiate repairing cellular membranes. Because of insufficient increase in the activity of the above enzymes, they cannot protect membranes in LA patients before antibiotics therapy. Proper diagnosis and treatment is very important because the isoprostanes may induce a signaling pathway that contributes to monocyte/ endothelial cell adhesion and potentially atherosclerosis. Antibiotic treatment leads to a decrease in the level of isoprostanes and in the activity of phospholipase $\mathrm{A}_{2}$ whereas the activity of PAF acethylhydrolase is enhanced. Therefore, the cellular homeostasis may not be quite reached, and the enhanced activity of PAF AH may lead to the development of metabolic disorders.

$\mathrm{LD}$ is generally responsive to antibiotic therapy; however, some patients develop an inflammatory arthritis that becomes refractory to antibiotic, and the musculoskeletal symptoms can continue for months after treatment. The pathomechanisms controlling the course of LD have not been fully explained, but it is known that the accompanying inflammatory state is characterized by stimulation of phagocytes which leads to enhanced generation of ROS and RNS [4] that cause NFKB activation in the endothelium. This, in consequence, leads to increased adhesion molecules expression (such as ICAM and VCAM-1), proinflammatory cytokines (IL1b, TNF- $\alpha$, IL-6, IL-12, IL-15) and chemokins (MCP-1, MIP) production. However, an increase in IFN- $\gamma$ concentration in macrophages leads to intense activity of indoleamine-pyrrole 2,3-dioxygenase, which additionally to ROS, regulates the activity of collagenases, elastase and stromelysin [23], which cause intensive degradation of the extracellular matrix. This process is faster due to oxidative modifications of the cell membrane phospholipids, which promote enhancing membrane permeability [18]. It enables spirochetes to penetrate various tissues, including articular cartilages. However, as shown in the presented study, the rate of arachidonic acid oxidation with isoprostanes generation at the sn-2 position is significantly higher than 
hydrolyzing free isoprostanes. Therefore, destabilization in the membrane structure connected with phospholipid peroxidation may be turned in the cross-linking resistant structure, especially that the oxidative modifications of membrane protein components may lead to the formation of intermolecular bridges. Such uncontrolled changes in the lipid-protein membrane structure may stick to the spirochete remnants that were found near cartilage in mice [24]. The finding of fluorescent deposits containing B. burgdorferi antigens in the knee entheses after antibiotic treatment has important implications for the pathogenesis of human LA. These remnants contain inflammatory potential and can stimulate macrophages to TNF-a production. Such debris could contribute to prolonged inflammatory responses in the joint after infectious spirochetes have been eradicated.

In humans, treatment-resistant LA has been attributed to autoimmunity because $B$. burgdorferi DNA can no longer be detected in the synovial fluid or synovectomy tissue [25]. However, infection-induced autoimmunity does not explain why this form of arthritis resolves over time, usually within four years. Release of foreign antigens could occur as a consequence of biomechanical stress at the enthese and contribute to inflammation at this site. Additionally, repeated oxidative stress formation may lead to slow degradation of $B$. burgdorferi components at these sites, which could contribute to the lag in symptom response in patients with disseminated LD.

\section{CONCLUSIONS}

1. In the course of LA, the level of binding 8-isoPGF is significantly enhanced, which may suggest that uncontrolled changes in the lipid status of some patients may render their Lyme arthritis unresponsive to antibiotics.

\section{Acknowledgements}

This study was supported by Grant No. N N401 315739 (Arrangement No. 3157/B/PO1/2010/39) from the National Science Centre in Krakow, Poland, and conducted with equipment from the Medical University of Białystok, Poland, purchased from the OP DEP 2007-2013, Priority Axis I.3, Contract No. POPW.01.03.00-20-008/09-MBN.

\section{Conflict of interest}

All authors declare they have no conflict of interests.

\section{REFERENCES}

1. Kean IR, Irvine KL. Lyme disease: aetiopathogenesis, factors for disease development and control. Inflammopharmacology 2013;21(2):101-11.

2. Nardelli DT, Callister SM, Schell RF. Lyme Arthritis: Current Concepts and a Change in Paradigm. Clin. Vaccine Immunol. 2008; 15: 21-34.

3. Guerau-de-Arellano M, Alroy J, Huber BT. Beta2 integrins control the severity of murine Lyme carditis. Infect Immun. 2005; 73: 3242-3250.
4. Suhonen J, Hartiala K, Tuominen-Gustafsson H, Viljanen MK. Borrelia burgdorferi-induced oxidative burst, calcium mobilization, and phagocytosis of human neutrophils are complement dependent. J Infect Dis. 2000; 181: 195-202.

5. Pancewicz SA, Skrzydlewska E, Hermanowska-Szpakowicz T, Zajkowska JM, Kondrusik M. Role of reactive oxygen species (ROS) in patients with erythema migrans, an early manifestation of Lyme borreliosis. Med Sci Monit. 2001; 7: 1230-1235.

6. Łuczaj W, Moniuszko A, Rusak M, Pancewicz S, Zajkowska J, Skrzydlewska E. Lipid peroxidation products as potential bioindicators of Lyme arthritis. Eur J Clin Microbiol Infect Dis. 2011; 30: 415-422.

7. Morrow JD, Chen Y, Brame CJ, Yang J, Sanchez SC, Xu J, Zackert WE, et al. The Isoprostanes: unique prostaglandin - like products of free radical initiated lipid peroxidation. Drug Metab Rev. 1999; 31: 117-139.

8. Seet RC, Lee CY, Lim EC, Tan JJ, Quek AM, Chong WL, et al. Oxidative damage in Parkinson disease: Measurement using accurate biomarkers. Free Radic Biol Med. 2010; 48: 560-566.

9. Milne GL, Yin H, Morrow JD. Human Biochemistry of the Isoprostane Pathway. J Biol Chem. 2008; 283: 15533-15537.

10. Coolen SA, van Buuren B, Duchateau G, Upritchard J, Verhagen H. Kinetics of biomarkers: biological and technical validity of isoprostanes in plasma. Amino Acids. 2005; 29: 429-436.

11. Li H, Lawson JA, Reilly M, Adiyaman M, Hwang S-W, Rokach J, FitzGerald GA. Quantitative high performance liquid chromatography/ tandem mass spectrometric analysis of the four classes of F2isoprostanes in human urine. Proc Natl Acad Sci USA. 1999; 96: 13381-13386.

12. Raatz SK, Bibus D, Thomas W, Kris-Etherton P. Total fat intake modifies plasma fatty acid composition in humans. J Nutr. 2001; 131: 231-234.

13. Bradford A, Atkinson J, Fuller N, Rand RP. The effect of vitamin E on the structure of membrane lipid assemblies. J Lipid Res. 2003; 44 : 1940-1945.

14. Blaho VA, Buczynski MW, Brown CR, Dennis EA. Lipidomic analysis of dynamic eicosanoid responses during the induction and resolution of Lyme arthritis. J Biol Chem. 2009; 284: 21599-21612.

15. Blaho VA, Mitchell WJ, Brown CR. Arthritis Develops but Fails to Resolve During Inhibition of Cyclooxygenase 2 in a Murine Model of Lyme Disease. Arthritis Rheum. 2008; 58: 1485-1495.

16. Roberts LJ2nd, Fessel JP. The biochemistry of the isoprostane, neuroprostane, and isofuran pathways of lipid peroxidation. Chem Phys Lipids. 2004; 128: 173-186.

17. Halliwell B, Lee CY. Using isoprostanes as biomarkers of oxidative stress: some rarely considered issues. Antioxid Redox Signal. 2010; 13: 145-156.

18. Halliwell B, Gutteridge JM. Free Radicals in Biology and Medicine. 3rd ed. New York, US, Oxford University Press, Inc., 1999.

19. Katsuki H, Okuda S. Arachidonic acid as a neurotoxic and neurotrophic substance. Prog Neurobiol. 1995; 46: 607-36.

20. Van den Berg JJM, Op den Kamp JAF, Lubin BH Kuypers FA. Conformational changes in oxidized phospholipids and their preferential hydrolysis by phospholipase A2: a monolayer study. Biochemistry. 1993; 32: 4962-4967.

21. McIntyre TM, Prescott SM, Stafforini DM. The emerging roles of PAF acetylhydrolase. J Lipid Res. 2009; 50: 255-259.

22. Ito S, Noguchi E, Shibasaki M, Yamakawa-Kobayashi K, Watanabe $\mathrm{H}$, Arinami T. Evidence for an association between plasma plateletactivating factor acetylhydrolase deficiency and increased risk of childhood atopic asthma. J Hum Genet. 2002; 47: 99-101.

23. Varga J, Yufit T, Hitraya E, Brown RR. Control of extracellular matrix degradation by interferon-gamma. The tryptophan connection. Adv Exp Med Biol. 1996; 398: 143-148.

24. Bockenstedt LK, Gonzalez DG, Haberman AM, Belperron AA. Spirochete antigens persist near cartilage after murine Lyme borreliosis therapy. J Clin Invest. 2012; 122: 2652-2660.

25. Li X, McHugh GA, Damle N, Sikand VK, Glickstein L, Steere AC. Burden and viability of Borrelia burgdorferi in skin and joints of patients with erythema migrans or Lyme arthritis. Arthritis Rheum. 2011; 63: 2238-2247. 